\title{
Cluster Head Selection in a Homogeneous Wireless Sensor Network Ensuring Full Connectivity with Minimum Isolated Nodes
}

\author{
Tapan Kumar Jain, Davinder Singh Saini, and Sunil Vidya Bhooshan \\ Department of Electronics and Communication Engineering, Jaypee University of Information Technology, \\ Waknaghat, Solan, Himachal Pradesh 173215, India
}

Correspondence should be addressed to Tapan Kumar Jain; tapan.jain@juit.ac.in

Received 2 December 2013; Accepted 28 June 2014; Published 12 August 2014

Academic Editor: Yanbiao Liao

Copyright (C) 2014 Tapan Kumar Jain et al. This is an open access article distributed under the Creative Commons Attribution License, which permits unrestricted use, distribution, and reproduction in any medium, provided the original work is properly cited.

\begin{abstract}
The research work proposes a cluster head selection algorithm for a wireless sensor network. A node can be a cluster head if it is connected to at least one unique neighbor node where the unique neighbor is the one that is not connected to any other node. If there is no connected unique node then the $\mathrm{CH}$ is selected on the basis of residual energy and the number of neighbor nodes. With the increase in number of clusters, the processing energy of the network increases; hence, this algorithm proposes minimum number of clusters which further leads to increased network lifetime. The major novel contribution of the proposed work is an algorithm that ensures a completely connected network with minimum number of isolated nodes. An isolated node will remain only if it is not within the transmission range of any other node. With the maximum connectivity, the coverage of the network is automatically maximized. The superiority of the proposed design is verified by simulation results done in MATLAB, where it clearly depicts that the total numbers of rounds before the network dies out are maximum compared to other existing protocols.
\end{abstract}

\section{Introduction}

Wireless sensor network (WSN) is a network of densely deployed large number of sensor nodes. WSNs are deployed to monitor physical events or the state of physical objects such as bridges in order to support appropriate reaction to avoid potential damages [1]. The nodes and the related protocols in a WSN should be designed to be extremely energy efficient as battery recharging may be impossible [2]. In direct communication WSN, the sensor nodes directly transmit their sensed data to the base station (BS) or sink without any coordination between the two. However, in cluster based WSNs, the network is divided into clusters. Each node exchanges its information only with its cluster head $(\mathrm{CH})$ which transmits the aggregated information to BS.

The most important phase of cluster-based routing protocols is the cluster head selection (CHS) procedure that ensures uniform distribution of energy among the sensors, and consequently increasing the lifespan of a sensor network [3]. Once the CHs are identified, they form a backbone network to periodically collect, aggregate, and forward data to the BS using the minimum energy (cost) routing. This method significantly enhances the network lifetime compared to other known methods. The major challenges include equal distribution of each cluster over the entire sensor network and the energy dissipation caused by the frequent information exchange between selected cluster head and nodes in the cluster in every setup phase of cluster formation $[4,5]$. If $\mathrm{CH}$ is selected on the basis of the concept of maximum number of nodes connected, then it may happen that one or more nodes are not connected to any of the selected cluster heads, even though they are in the transmission range. Such nodes are called the isolated nodes. The proposed algorithm deals with the cluster head selection based on the unique node concept. A unique node is the one which is not connected to any other cluster heads. The current paper describes CHS using two other parameters as well, namely, number of neighboring nodes and the residual energy of the node. 
TABle 1: Comparison of cluster head selection techniques.

\begin{tabular}{lcccc}
\hline Authors (year) & Residual energy & Number of nodes & Unique node & Distance from BS \\
\hline Azad and Sharma [6] (2013) & Yes & Yes & No & No \\
Lee et al. [7] (2006) & Yes & No & No & Yes \\
Chan and Perrig [8] (2004) & No & No & No & No \\
Heinzelman et al. [2] (2002) & Yes & No & No & Yes \\
Ferdous and Dey [9] (2010) & No & Yo & No & No \\
Mini et al. [10] (2012) & No & No & No & Yes \\
Lee et al. [11] (2014) & No & & & \\
\hline
\end{tabular}

The rest of the paper is organized as follows. Section 2 describes the related work of routing in WSN and emphasizes on existing $\mathrm{CH}$ selection methods. Section 3 explains the system model and assumptions to design the algorithm. Section 4 gives $\mathrm{CH}$ selection algorithm in detail along with its mathematical model. The flowchart is also used to depict the proposed algorithm. Section 5 gives the simulation results done in MATLAB and compares the results with existing methods. Section 6 concludes and proposes the work for future.

\section{Related Work}

WSNs employ low-cost, densely deployed, tiny electronic nodes connected to each other via wireless communication [12]. The most power-consuming activity of a sensor node is radio communication which must be kept as low as possible. In order to reduce the amount of traffic in the network, we build clusters of sensor nodes as proposed in [12-14].

Clustering is a useful mechanism in wireless sensor networks that helps to combat with scalability problems, and, if combined with in-network data aggregation, may increase the energy efficiency of the network [15]. $\mathrm{CH}$ is the main entity in a wireless sensor network, and all the responsibility for the data aggregation and communication lies with this single entity [2]. CH should be chosen in such a way such that the coverage of the network is a maximum. Coverage is regarded as one of the important qualities of service (QoS) parameter of a WSN to evaluate its monitoring capability [8].

Sensor node consumes most of its power on communication, especially when it needs to transmit its data to the BS which is located far away from the WSN [2, 8, 16-18]. To overcome this problem, routing protocols for WSN have been developed such as weighted clustering [17], hierarchal clustering $[2,8,16]$, and dynamic clustering [18] algorithms. Hierarchical cluster based routing protocols (e.g., ACE [8], HEED [16], and LEACH-C [2]) are worth mentioning.

ACE [8] successfully distributes clusters uniformly over the network but suffers from its unawareness of residual energy in cluster-heads candidates, which results in electing a cluster head with low energy level. The other disadvantage of ACE strictly draws a line between nodes that can be a cluster head and the ones who cannot. In some cases, this assumption may be unrealistic, especially when all the nodes within a cluster have low power resources. The current research work eliminates both the problems, by considering the residual energy while selecting the $\mathrm{CH}$. The proposed work takes a homogeneous network in which all the nodes are the same with equal power and have equal opportunity of becoming a $\mathrm{CH}$.

In the research article [10], the authors talk about $M$ connectivity in which each mote is connected to at least $M$ other motes in the same cover. But it may happen that a node in the network is not connected to any other node at all which does not ensure a completely connected network.

The related researches in the literature for cluster-head selection in WSN are many. The brief details of some of them [2, 6-11] are depicted in Table 1.

Table 1 inspires the authors to come up with a CHS algorithm that selects any $\mathrm{CH}$ on the basis of any unique note connected to it, the residual energy, and the number of nodes connected to it.

\section{Methodology}

\subsection{System Model and Assumptions}

3.1.1. Assumptions. The following assumptions have been made for the proposed network.

(1) The nodes are homogenous with initial energy of $1 \mathrm{~J}$.

(2) The nodes transmit the data to the BS in multiple hops.

(3) The hops are determined based upon distance from the BS.

(4) The sensors used have transmitting range of 100$150 \mathrm{~m}$ (outdoor) and 50-75 $\mathrm{m}$ (indoor). If $R_{t}(150 \mathrm{~m})$ and $R_{s}(75 \mathrm{~m})$ represents transmission and sensing range, respectively, it is assumed that $R_{t} \geq 2 R_{s}$ as given in [5]. If there is one more sensor within the transmission range of a sensor, then the sensed information is the same for both the sensors.

(5) The nodes are randomly deployed.

(6) Sensor nodes are stationary.

(7) BS $\left(n_{0}\right)$ is fixed and installed somewhere in the middle of the network $(250,250)$.

(8) The nodes are proactive; that is, they transfer data to the $\mathrm{BS}$ in periodic intervals.

(9) The CHs are selected initially at the onset of the network. The $\mathrm{CH}$ is reselected or rotated as per the proposed algorithm if the energy of the $\mathrm{CH}$ falls below the threshold. 


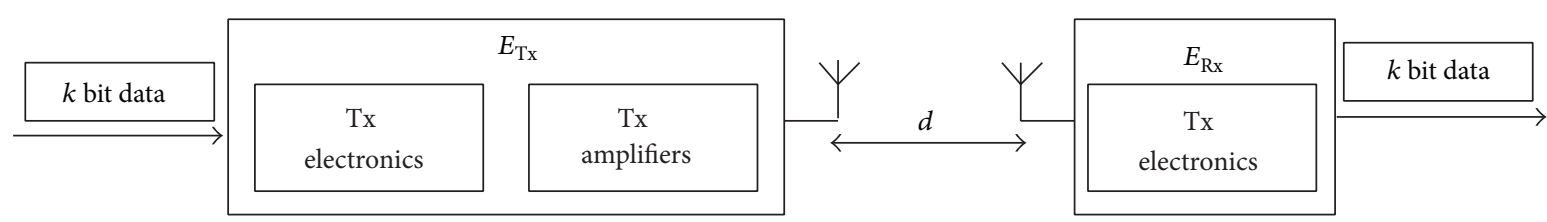

Figure 1: First-order energy model.

TABLE 2: Radio characteristics.

\begin{tabular}{lc}
\hline Operation & Energy dissipated \\
\hline Transmitter electronics $\left(E_{\text {Tx-elec }}\right)$ & \\
Receiver electronics $\left(E_{\mathrm{Rx} \text {-elec }}\right)$ & $50 \mathrm{~nJ} / \mathrm{bit}$ \\
$E_{\mathrm{Tx}-\text { elec }}=E_{\mathrm{Rx} \text {-elec }}=E_{\mathrm{elec}}$ & \\
\hline Transmit amplifier $\left(\varepsilon_{\mathrm{amp}}\right)$ & $100 \mathrm{pJ} / \mathrm{bit} / \mathrm{m}^{2}$ \\
\hline
\end{tabular}

3.1.2. System Model. The use of clusters for transmitting data limits the number of nodes that transmit to BS avoiding transmission to short distances. In the first-order model [2] shown in Figure 1, different assumptions about the radio characteristics, like energy dissipation in the transmitting and receiving nodes, path loss exponent, and so forth, will change the advantages of different protocols. Here, we have assumed a simple model where the radio dissipates $50 \mathrm{~nJ} / \mathrm{bit}$ to run the transmitter/receiver circuitry. Further, it requires $100 \mathrm{pJ} / \mathrm{bit} / \mathrm{m}^{2}$ for transmit amplifier to achieve an acceptable $E b / N_{o}$ as depicted in Table 2 . These parameters are slightly better than the current state of the art in radio design.

The impact of energy loss due to channel transmission is also considered. Thus, to transmit a $k$-bit message over distance $d$ using our radio model, the energy required is given by

$$
\begin{aligned}
& E_{\mathrm{Tx}}(k, d)=E_{\mathrm{Tx} \text {-elec }}(k)+E_{\mathrm{Tx} \text {-amp }}(k, d), \\
& E_{\mathrm{Tx}}(k, d)=E_{\text {elec }} * k+\varepsilon_{\mathrm{amp}} * k * d^{2}, \quad d<d_{0} .
\end{aligned}
$$

The received energy can be calculated by

$$
\begin{aligned}
& E_{\mathrm{Rx}}(k)=E_{\mathrm{Rx}-\text { elec }}(k), \\
& E_{\mathrm{Rx}}(k)=E_{\text {elec }} * k .
\end{aligned}
$$

Receiving a message is not a low cost operation. The protocols try to minimize transmit distance along with transmitting and receiving operations for each message.

The notations used in the paper are detailed in the Abbreviations section.

\section{Proposed Clustering Algorithm}

On the basis of location of various nodes, the proposed algorithm identifies the clusters and $\mathrm{CHs}$. The $\mathrm{CH}$ are chosen on the basis of unique connected nodes, maximum number of neighbor nodes, and the residual energy. After $\mathrm{CH}$ identification, the data transmission from a specific node
TABLE 3: Neighbor information details for the first hop.

\begin{tabular}{cccc}
\hline$I$ & $n_{i}$ & count $_{i}$ & $N b_{i}$ \\
\hline 0 & $n_{0}$ & 3 & $n_{1} n_{2} n_{3}$ \\
1 & $n_{1}$ & 3 & $n_{0} n_{2} n_{7}$ \\
2 & $n_{2}$ & 6 & $n_{0} n_{1} n_{4} n_{5} n_{7} n_{10}$ \\
3 & $n_{3}$ & 5 & $n_{0} n_{11} n_{12} n_{13} n_{14}$ \\
\hline
\end{tabular}

is done using $\mathrm{CHs}$ till it reaches BS. Each $\mathrm{CH}$ combines the data collected from its connected nodes and performs the data aggregation that reduces the amount of data to be transmitted. The aggregated data is sent to the BS through the intermediate $\mathrm{CHs}$ as per routing table established earlier. The stepwise algorithm is described in the following section.

Step 1. $N$ number of motes is randomly deployed in the region to be sensed. The $\mathrm{BS}$ is given in the id of 0 and it is manually located on the network field:

$$
N=\left\{n_{1}, n_{2}, n_{3}, \ldots, n_{N}\right\} .
$$

Step 2. Calculate the set of neighbor nodes $N b_{i}$ and the number of neighbor nodes count ${ }_{i}$ for the $i$ th node $n_{i}$ on the basis of the transmission range as depicted in Table 3:

$$
\text { function }\left[\operatorname{count}_{i}, N b_{i}\right]=\text { neighbor_info }\left(n_{i}\right) \text {. }
$$

The function neighbor_info returns all the neighboring nodes of a node $n_{i}$.

The distance between a given sensor $n_{i}$ and $n_{j}$ is given by

$$
\begin{gathered}
d_{i j}=\sqrt{\left(x_{i}-x_{j}\right)^{2}+\left(y_{i}-y_{j}\right)^{2}}, \\
i=1,2, \ldots, N, \quad j=1,2, \ldots, N ; \\
\left\{N b_{i} \mid d_{i j}<R_{t} \forall j, \quad j \in N\right\} .
\end{gathered}
$$

$N b_{i}=\left\{n_{1}, n_{2}, n_{3}, \ldots, n_{n}\right\}$; set of neighbor nodes IDs within the transmitting range $\left(R_{t}\right)$. neighbor_info function is repeated for all $N$ motes and the BS. The function neighbor_info gives the result as shown in Figure 2.

Step 3. BS always behaves as the $\mathrm{CH}$. $\mathrm{CH}$ selection (CHS) process starts from BS. BS transmits the $\mathrm{CH}$ selection message (CHS_msg) to all the neighboring nodes. Nodes having residual energy more than the threshold energy are eligible to become a CH. Step 3 is depicted in Figure 3.

All_neighbours function returns the set of all the neighboring nodes [Neighbours] of $N b_{i}$. 


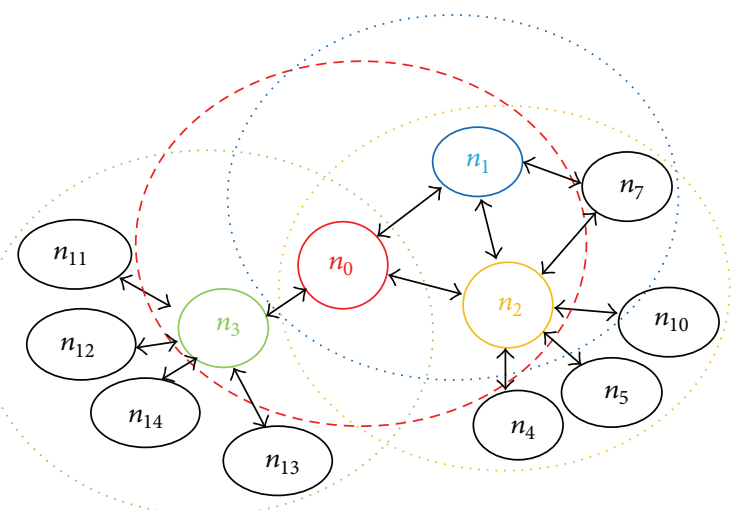

FIGURE 2: Network model: nodes with transmitting range.

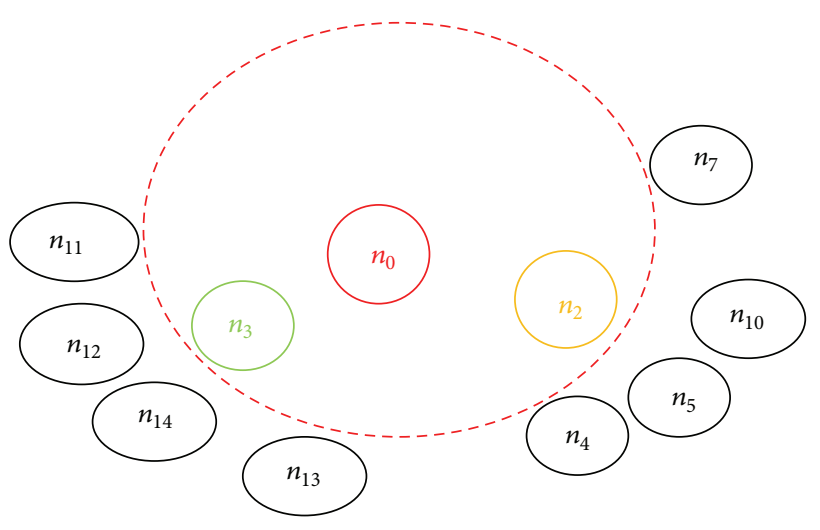

Figure 3: Step 3, Neighbors.

Step 3_A. This step is only applicable when the base station determines its neighbor and set the base station position according to the below equation to minimize the base station to neighbor node distance to save the energy and reduce the synchronization time; $\sum_{i=1}^{\text {count }} d_{i} \rightarrow \min$. The optimal base station coordinates are then given by

$$
\left(x_{0}, y_{0}\right)=\arg \min \min _{(x, y)} \sum_{i=1}^{N} \sqrt{\left(x_{i}-x\right)^{2}+\left(y_{i}-y\right)^{2}} .
$$

The minimum is obtained by setting the partial derivatives to zero:

$$
\frac{\partial}{\partial x} \sum_{i=1}^{\text {count }_{i}} d_{i}=\frac{\partial}{\partial y} \sum_{i=1}^{\text {count }_{i}} d_{i}=0 \quad \text { for } x=x_{o}, y=y_{o} .
$$

The partial derivatives are

$$
\begin{aligned}
\frac{\partial}{\partial x} \sum_{i=1}^{\text {count }_{i}} d_{i} & =\sum_{i=1}^{\text {count }_{i}} \frac{\partial}{\partial x} d_{i}=\sum_{i=1}^{\text {count }_{i}} \frac{x_{i}-x}{d_{i}}, \\
\frac{\partial}{\partial y} \sum_{i=1}^{\text {count }_{i}} d_{i} & =\sum_{i=1}^{\text {count }_{i}} \frac{\partial}{\partial y} d_{i}=\sum_{i=1}^{\text {count }_{i}} \frac{y_{i}-y}{d_{i}} .
\end{aligned}
$$

By using the vector notations, the vector pointing to the location of the $i$ th sensor node is $n_{i}=\left(x_{i}, y_{i}\right)$, and the distance

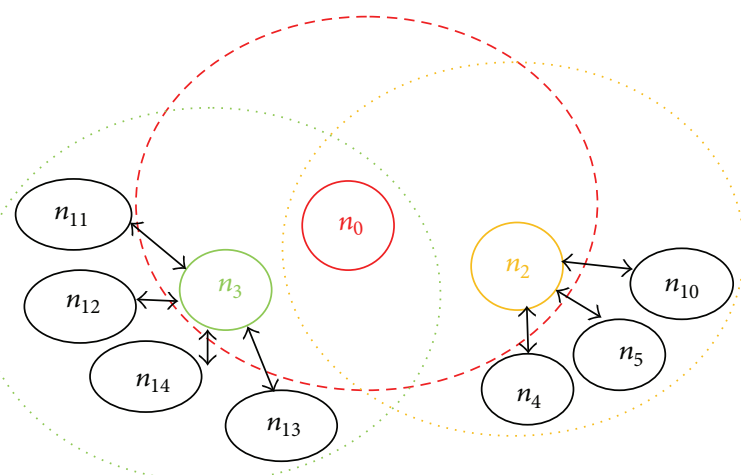

Figure 4: Step 4, $N u b_{i}$.

TABLE 4: Unique neighbor details for the first hop.

\begin{tabular}{cccccc}
\hline$i$ & $n_{i}$ & count $_{i}$ & $N b_{i}$ & ucount $_{i}$ & $N u b_{i}$ \\
\hline 1 & $n_{1}$ & 3 & $n_{0} n_{2} n_{7}$ & 0 & - \\
2 & $n_{2}$ & 6 & $n_{0} n_{1} n_{4} n_{5} n_{7} n_{10}$ & 3 & $n_{4} n_{5} n_{10}$ \\
3 & $n_{3}$ & 5 & $n_{0} n_{11} n_{12} n_{13} n_{14}$ & 4 & $n_{11} n_{12} n_{13} n_{14}$ \\
\hline
\end{tabular}

vector between the sensor node and sink is $d_{i}=n_{i}-s=$ $\left(\left(x_{i}, x\right),\left(y_{i}, y\right)\right)$. Let $e_{i}$ be the unit vector from $i$ th sensor node towards the nearest sink (the orientation vector); that is,

$$
e_{i}=\frac{d_{i}}{d}=\frac{1}{d_{i}}\left(\left(x_{i}, x\right),\left(y_{i}, y\right)\right) .
$$

Using the above three equations $r=\sum_{i=1}^{\text {count }_{i}} e_{i}=0$ for $s=\left(x_{0}, y_{0}\right)$, that is, the average distance is minimized if the resultant $r$ of the orientation vector is zero,

$$
\text { Function [Neighbours] = all_neighbours }\left(N b_{i}\right) \cup N b_{i} \text {. }
$$

Neighbours for $N b_{0}=\left\{n_{1} n_{2} n_{3}\right\} \cup\left\{n_{0} n_{2} n_{7}\right\} \cup$ $\left\{\begin{array}{lllllllll}n_{0} & n_{1} & n_{4} & n_{5} & n_{7} & n_{10}\end{array}\right\} \quad \cup \quad\left\{\begin{array}{llllll}n_{0} & n_{11} & n_{12} & n_{13} & n_{14}\end{array}\right\} \quad=$ $\left\{\begin{array}{lllllllllllll}n_{1} & n_{2} & n_{3} & n_{4} & n_{5} & n_{7} & n_{10} & n_{11} & n_{12} & n_{13} & n_{14}\end{array}\right\}$ except $n_{0}$.

In Neighbours the CH id or initially BS ID is excluded.

Step 4. The function unique_neighbor_info returns the unique nodes connected to a node. The unique nodes are the ones which are not connected to any other nodes in the $(i+1)$ th hop as shown in Figure 4. Table 4 gives the details of the unique neighbors for first hop (see Algorithm 1).

A flag is set for all the $N b_{i}$ nodes appearing in the set Neighbours for $\mathrm{Nb}_{i}$.

Step 5. Consider the unflagged elements of Neighbours.

The function set_of_neighbours returns $N N b_{i}$ which is the set of all the neighbours of members of $N b_{i}$

$$
\text { Function }\left[N\left(N b_{i}\right)\right]=\text { set_of neighbours }\left(N b_{i}\right) \text {. }
$$

$N\left(N b_{i}\right)$ is depicted in Table 4. 


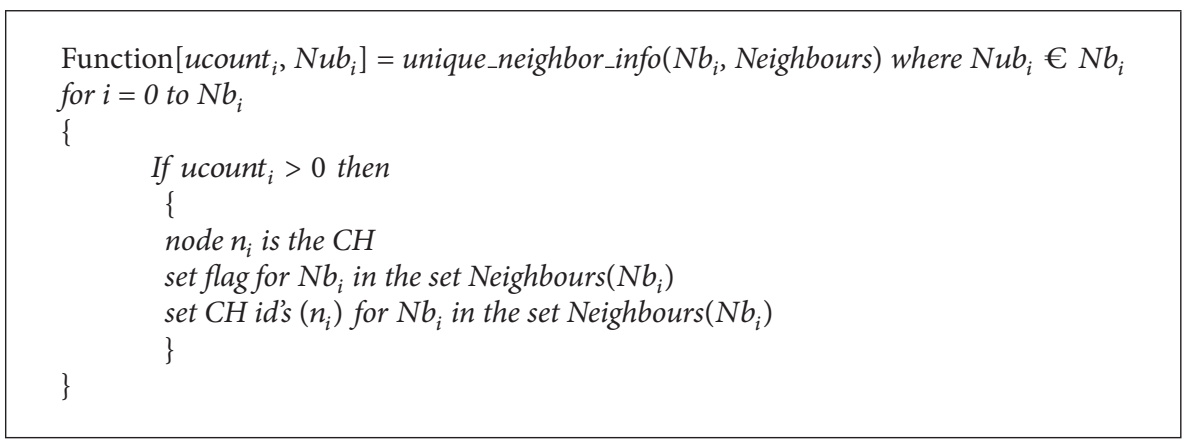

Algorithm 1

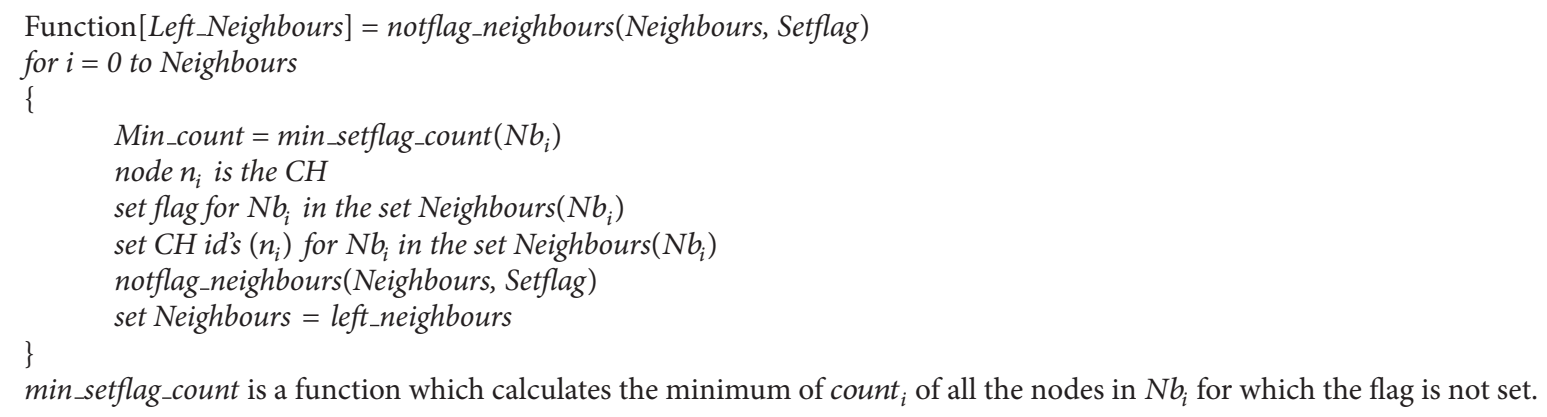

Algorithm 2

In Table $5, N\left(N b_{0}\right)$ is

$$
\begin{aligned}
& i=0 \\
N\left(N b_{i}\right)= & N\left(N b_{0}\right) \\
= & N\left(\begin{array}{lll}
n_{1} & n_{2} & n_{3}
\end{array}\right) \\
= & N b_{1}, N b_{2}, N b_{3} \\
= & \left\{\begin{array}{lll}
n_{0} & n_{2} & n_{7}
\end{array}\right\},\left\{\begin{array}{llllll}
n_{0} & n_{1} & n_{4} & n_{5} & n_{7} & n_{10}
\end{array}\right\}, \\
& \left\{\begin{array}{lllll}
n_{0} & n_{11} & n_{12} & n_{13} & n_{14}
\end{array}\right\} .
\end{aligned}
$$

The Left_Neighbours contains the nodes whose cluster ID flag is not set by unique count (see Algorithm 2).

Step 6. Steps 3 to 5 are repeated till all the nodes are covered by elected $\mathrm{CH}$. All nodes should have a path up to BS through single or multiple hops depending upon the distance of the node from the BS.

Step 7. After clustering, if in the process of transmission of data, one of the $\mathrm{CH}$ die out, then the $\mathrm{CH}$ at the previous hop comes to know about it since the data from the dead $\mathrm{CH}$ did not reach it. Suppose the $\mathrm{CH}$ at $n$th hop dies out; then in such

\begin{tabular}{|c|c|c|c|}
\hline$N N b_{i}$ & $n_{i}$ & & $N b_{i}$ \\
\hline & $n_{0}$ & $N b_{0}$ & $n_{1} n_{2} n_{3}$ \\
\hline$N N b_{0}$ & & $N b_{1}$ & $n_{0} n_{2} n_{7}$ \\
\hline$N N b_{0}$ & & $\mathrm{Nb}_{2}$ & $n_{0} n_{1} n_{4} n_{5} n_{7} n_{10}$ \\
\hline$N N b_{0}$ & & $\mathrm{Nb}_{3}$ & $\begin{array}{lllll}n_{0} & n_{11} & n_{12} & n_{13} & n_{14}\end{array}$ \\
\hline
\end{tabular}
a case the clustering algorithm is repeated after $(n-1)$ th hop for the entire network.

Step 8. If the residual energy of the $\mathrm{CH}$ becomes less than the threshold energy $\left(E_{\mathrm{th}}\right)$, then the $\mathrm{CH}$ selection process
TABLE 5: Neighbor information details for the first hop.

needs to be reinstantiated. Suppose the residual energy of the $\mathrm{CH}$ at $n$th hop is less than the threshold; then the clustering algorithm is repeated after $(n-1)$ th hop for that particular path.

Step 9. Once the routing path is established the data transmits through multihop. Each $\mathrm{CH}$ combines the data collected from its connected nodes through data aggregation.

Data aggregation is any process in which information is gathered and expressed in a summary form. Many research papers [19] have shown that aggregation at the $\mathrm{CH}$ considerably reduces the amount of data routed through the network, increasing the throughput and extending the lifetime of the sensor networks.

Data aggregation also solves the purpose of estimating a missing value from a sensor [20]. Sometimes it may happen that data from one of the sensors did not reach the cluster head, in such cases the Jackknife estimate can be used to 


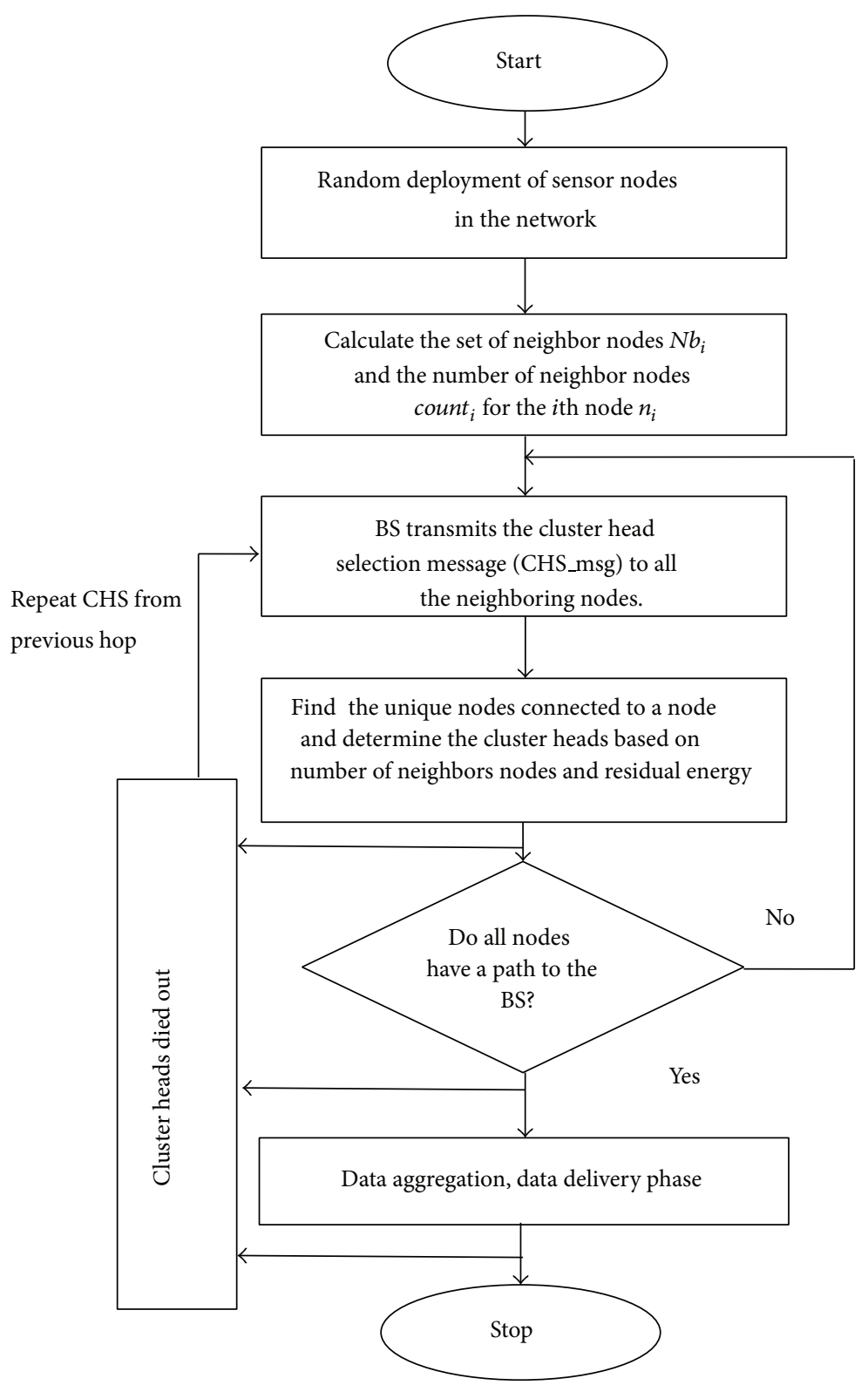

FIGURE 5: Flowchart.

predict the value of the sensed parameter. It also performs fault tolerance. If the data received from one of the sensors does not match the estimated value, then accordingly the correction is made.

After aggregating the data from all the nodes in the cluster, the $\mathrm{CH}$ sends the data to the next $\mathrm{CH}$ or the $\mathrm{BS}$ as the case may be. The flowchart of the complete proposed algorithm is shown in Figure 5.

\section{Results and Discussions}

Simulations are carried out to evaluate the proposed algorithm in MATLAB. Simulation is done with nodes placed randomly using uniform distribution throughout the network of dimension $500 \mathrm{~m} \times 500 \mathrm{~m}$. The BS is located at $x=250$ and $y=250$. The simulation parameters considered are as described in Table 5. The transmission cluster radius is taken as $150 \mathrm{~m}$ and initial threshold energy $E_{\text {th }}=E_{0} / 2$.

The proposed algorithm is evaluated using the following measures: network lifetime and connectivity. After some rounds of transmission, when the residual energy of all the nodes approaches to $E_{\text {th }}$ then the network adaptively reduces the value of $E_{\mathrm{th}}$, thus increasing the network lifetime. Data aggregation at the cluster heads further enhances the network lifetime by reducing the size of the data to be transmitted by the nodes. When the cluster head residual energy is less than or equal to the threshold energy, only then the cluster head selection algorithm is carried out for $n-1$ hops. Thus, the proposed algorithm ensures that energy is not wasted in cluster head selection for every round. The network 
TABLE 6: Simulations parameters values.

\begin{tabular}{lc}
\hline Notation & Meaning \\
\hline$N$ & 50,100 \\
$E_{0}$ & Initial node energy $(1 \mathrm{~J})$ \\
$n_{0}$ & $(250,250)$ location \\
$n_{i}$ & Node id of $i$ th node \\
$A$ & $(0,0)$ to $(500,500)=250000 \mathrm{~m}^{2}$ \\
$R_{s}$ & Point sensor \\
$R_{t}$ & $100 \mathrm{~m}, 150 \mathrm{~m}$ \\
$E_{r}$ & $E_{0}-E_{\text {processing }}-E_{\mathrm{DA}}-E_{\text {sensing }}$ \\
$E_{\text {th }}$ & $0.5 \mathrm{~J}$ initially till it reaches $0.1 \mathrm{~J}$ \\
\hline
\end{tabular}

connectivity is effectively handled by the algorithm. The cluster heads connect all the nodes of the network. The proposed algorithm ensures that none of the unique nodes are left behind without being connected to the network; hence, the possibility of having isolated nodes is minimized.

The proposed algorithm is compared with DTE (direct transmission energy) [21], Leach (low energy adaptive clustering hierarchy) [2], and multihop routing, ACE [8]. For each protocol, nodes are randomly deployed by generating random coordinates using uniform distribution. For each protocol, 100 iterations were performed and the result is their average. We performed simulation on 50 and 100 nodes. The values of the simulation parameters are shown in Table 6 . The simulation results and comparison between the different $\mathrm{CHS}$ protocols are depicted in Figure 6. Data aggregation energy is not considered in the simulation.

FND (first node die) corresponds to the number of rounds and the networks runs before the first deployed sensing node dies out.

$10 \%$ die out means that $10 \%$ of the sensing nodes deployed die out.

$90 \%$ die out means that $90 \%$ of the sensing nodes deployed die out.

The above results clearly show that the proposed algorithm gives better results as compared to existing methods. In this case, the stability period is increased because the $\mathrm{CH}$ is rotating, only when the threshold value reaches $0.1 \mathrm{~J}$.

\section{Conclusion and Future Work}

The proposed algorithm for $\mathrm{CH}$ selection in a WSN using unique node concept has many advantages. The latency in transmitting the data in a single hop is much more than in the proposed multihop wireless sensor network. Each node in the network transmits the data to $\mathrm{CH} / \mathrm{BS}$ closest to it. The $\mathrm{CH}$ in turn transmits the data to the next $\mathrm{CH}$, if required, to reach the BS. If the $\mathrm{CH}$ is selected on the basis of the concept of maximum number of nodes connected, then it may happen that one or more unique nodes are not connected to any of the selected CHs. Thus, this algorithm deals with the $\mathrm{CH}$ selection based on the unique node concept.

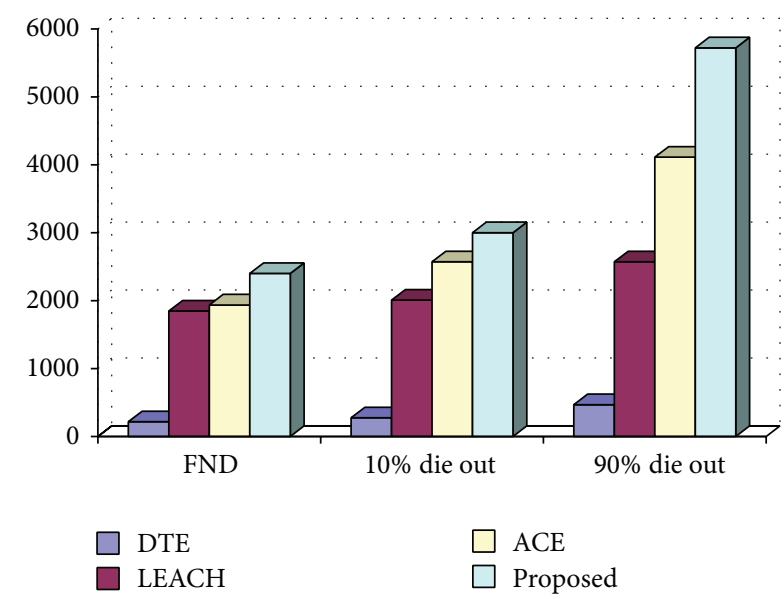

FIGURE 6: Simulations results and comparison Chart.

In the proposed algorithm there is no possibility of having any outlier, as all the unique nodes are connected to some or the other CHs. Adaptability is well taken care of. After clustering, if in the process of transmission of data, one of the $\mathrm{CHs}$ dies out, then the $\mathrm{CH}$ at the previous hop comes to know about it since the data from the dead CH did not reach it and the clustering algorithm is repeated after $(n-1)$ th hop for the entire network. If the residual energy of the $\mathrm{CH}$ becomes less than the threshold energy then the $\mathrm{CH}$ section process is reinstantiated after $(n-1)$ th hop for that particular path. Table 6 clearly depicts that the proposed clustering algorithm increases the network lifetime. In wireless sensor networks, the communication cost is often several orders of magnitude larger than the computation cost; thus, the $\mathrm{CHs}$ perform data aggregation to reduce the amount of data to be transmitted. The proposed work is a 1-connected network. If any node fails then the algorithm is run again to perform clustering a-fresh. The drawback is that a lot of energy is wasted in reclustering. In the future, the authors plan to develop a Q-connected network ensuring full connectivity with minimum number of isolated nodes. In the future, the authors plan to incorporate the proposed algorithm for heterogeneous wireless sensor network in which the $\mathrm{CH}$ will have more power than the connected nodes to perform data aggregation.

\section{Abbreviations}

$N: \quad$ Total nodes in the network

$E_{0}$ : Initial node energy (1 J)

$n_{0}: \quad$ Node id of BS

$n_{i}$ : $\quad$ Node id of $i$ th node

$K: \quad$ Number of bits in one packet

$E_{\text {th }}$ : Energy threshold value at which the $\mathrm{CH}$ selection restarts

$d_{i j}$ : $\quad$ Distance between $i$ th node to $j$ th node

$E_{\mathrm{DA}}$ : Data aggregation energy

$N_{i}$ : Set of neighboring nodes of $i$ th node

$R_{s}$ : Sensing range

$R_{t}$ : Transmitting range

$\mathrm{CH}_{\mathrm{id}}$ : Cluster head ID's for each node 
$E_{r}: \quad$ Residual energy $<E_{0}$

$N b_{i}$ : ith hop neighbor of BS or neighbor of nodes in $(i-1)$ th hop

Count $_{i}$ : The number of neighbors of $i$ th node

$E_{\text {sensing: }}$ Sensing energy.

\section{Conflict of Interests}

The authors declare that there is no conflict of interests regarding the publication of this paper.

\section{Acknowledgment}

The authors are sincerely thankful to the potential reviewers for their valuable comments and suggestions to improve the quality of the paper.

\section{References}

[1] A. Ali, A. Khelil, F. K. Shaikh, and N. Suri, "Efficient predictive monitoring of wireless sensor networks," International Journal of Autonomous and Adaptive Communications Systems, vol. 5, no. 3, pp. 233-254, 2012.

[2] W. B. Heinzelman, A. P. Chandrakasan, and H. Balakrishnan, "An application-specific protocol architecture for wireless microsensor networks," IEEE Transactions on Wireless Communications, vol. 1, no. 4, pp. 660-670, 2002.

[3] S. H. Kang and T. Nguyen, "Distance based thresholds for cluster head selection in wireless sensor networks," IEEE Communications Letters, vol. 16, no. 9, pp. 1396-1399, 2012.

[4] J.-S. Kim, S.-Y. Choi, S.-J. Han et al., "Alternative cluster head selection protocol for energy efficiency in wireless sensor networks," in Software Technologies for Future Dependable Distributed Systems, pp. 159-163, Tokyo, Japan, March 2009.

[5] T. Jain, "Wireless environmental monitoring system (WEMS) using data aggregation in a bidirectional hybrid protocol," in Proceedings of the 6th International Conference on Information Systems, Technology and Management (ICISTM '12), pp. 414420, Grenoble, France, March 2012.

[6] P. Azad and V. Sharma, "Cluster head selection in wireless sensor networks under fuzzy environment," ISRN Sensor Networks, vol. 2013, Article ID 909086, 8 pages, 2013.

[7] H. S. Lee, K. T. Kim, and H. Y. Youn, "A new cluster head selection scheme for long lifetime of wireless sensor networks," in Computational Science and Its Applications-ICCSA 2006, vol. 3983 of Lecture Notes in Computer Science, pp. 519-528, Springer, Berlin, Germany, 2006.

[8] H. Chan and A. Perrig, "ACE: an emergent algorithm for highly uniform cluster formation," in Proceedings of the 1st European Workshop on Sensor Networks (EWSN '04), pp. 154-171, Berlin, Germany, 2004.

[9] J. Ferdous and T. Dey, "A comprehensive analysis of CBCDACP in wireless sensor networks," Journal of Communications, vol. 5, no. 8, pp. 627-636, 2010.

[10] S. Mini, S. K. Udgata, and S. L. Sabat, " $M$-connected coverage problem in wireless sensor networks," ISRN Sensor Networks, vol. 2012, Article ID 85802, 9 pages, 2012.

[11] J.-Y. Lee, K. Jung, H. Jung, and D. Lee, "Improving the energy efficiency of a cluster head election for wireless sensor networks," International Journal of Distributed Sensor Networks, vol. 2014, Article ID 305037, 6 pages, 2014.
[12] Y. E. M. Hamouda and C. Phillips, "Adaptive sampling for energy-efficient collaborative multi-target tracking in wireless sensor networks," IET Wireless Sensor Systems, vol. 1, no. 1, pp. 15-25, 2011.

[13] S. Ganesh and R. Amutha, "Efficient and secure routing protocol for wireless sensor networks through snr based dynamic clustering mechanisms," IEEE Journal of Communication and networks, vol. 15, no. 4, pp. 422-429, 2013.

[14] G. Pei and C. Chien, "Low power TDMA in large wireless sensor networks," in Proceedings of the Military Communications Conference, pp. 347-351, 2001.

[15] L. Buttyán and T. Holczer, "Private cluster head election in wireless sensor networks," in Proceedings of the IEEE 6th International Conference on Mobile Adhoc and Sensor Systems (MASS '09), pp. 1048-1053, Macau, China, October 2009.

[16] O. Younis and S. Fahmy, "HEED: a hybrid, energy-efficient, distributed clustering approach for ad hoc sensor networks," IEEE Transactions on Mobile Computing, vol. 3, no. 4, pp. 366379, 2004.

[17] M. Chatterjee, S. K. Das, and D. Turgut, "WCA: a weighted clustering algorithm for mobile ad hoc networks," Journal of Cluster Computing, vol. 5, no. 2, pp. 193-204, 2002.

[18] W. P. Chen, J. C. Hou, and L. Sha, "Dynamic clustering for acoustic target tracking in wireless sensor networks," IEEE Transactions on Mobile Computing, vol. 3, no. 3, pp. 258-271, 2004.

[19] M. Watfa, W. Daher, and H. Al Azar, "A sensor network data aggregation technique," International Journal of Computer Theory and Engineering, vol. 1, no. 1, pp. 19-26, 2009.

[20] K. Fan, S. Liu, and P. Sinha, "Structure-free data aggregation in sensor networks," IEEE Transactions on Mobile Computing, vol. 6, no. 8, pp. 929-942, 2007.

[21] Q. Yang, X. Yang, Y. Wang, and A. Dou, "A strategy based on cooperative transmission for minimizing delivery delay in WSN," International Journal of Distributed Sensor Networks, vol. 2013, Article ID 286237, 10 pages, 2013. 

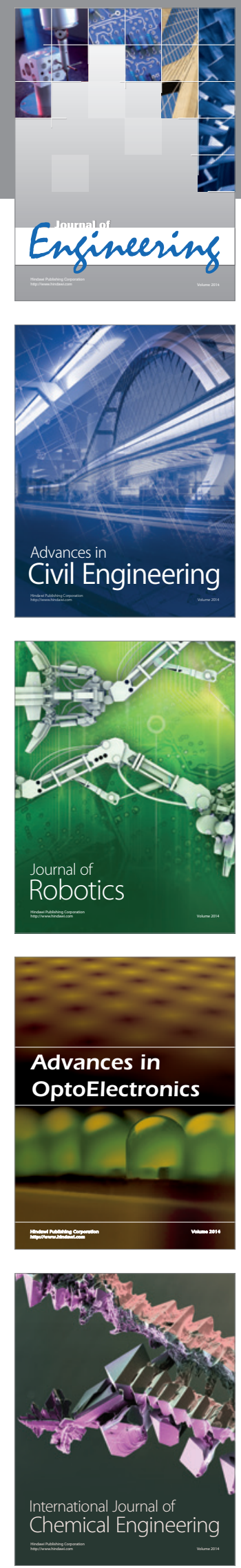

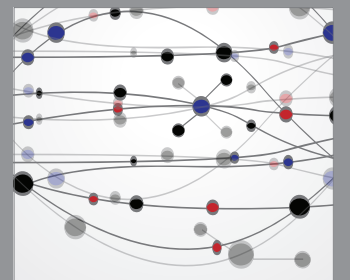

The Scientific World Journal
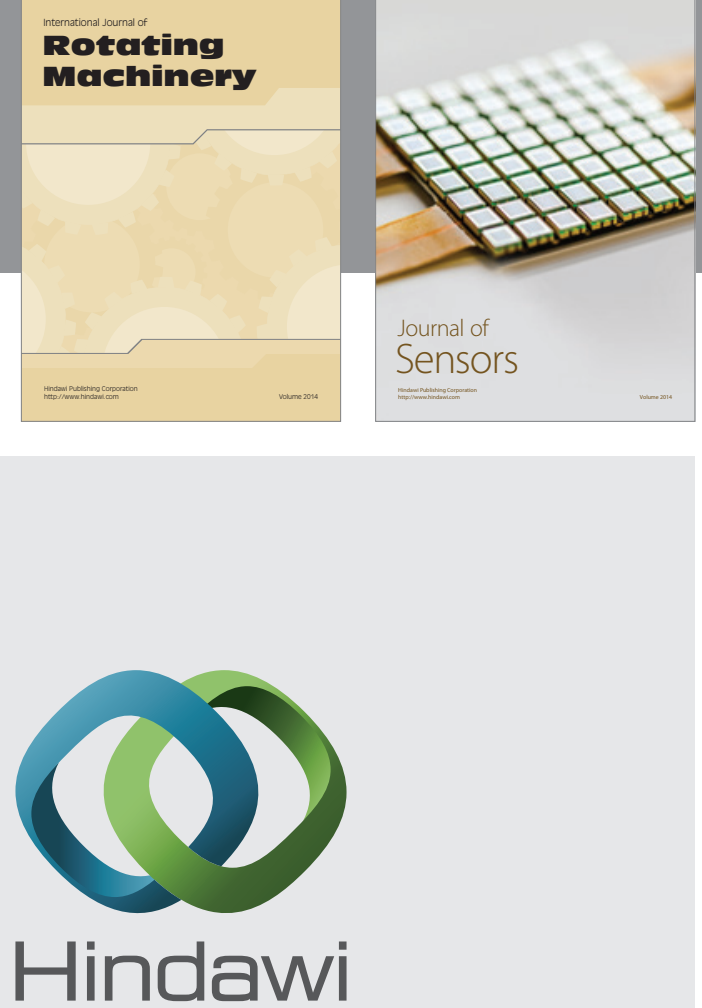

Submit your manuscripts at http://www.hindawi.com
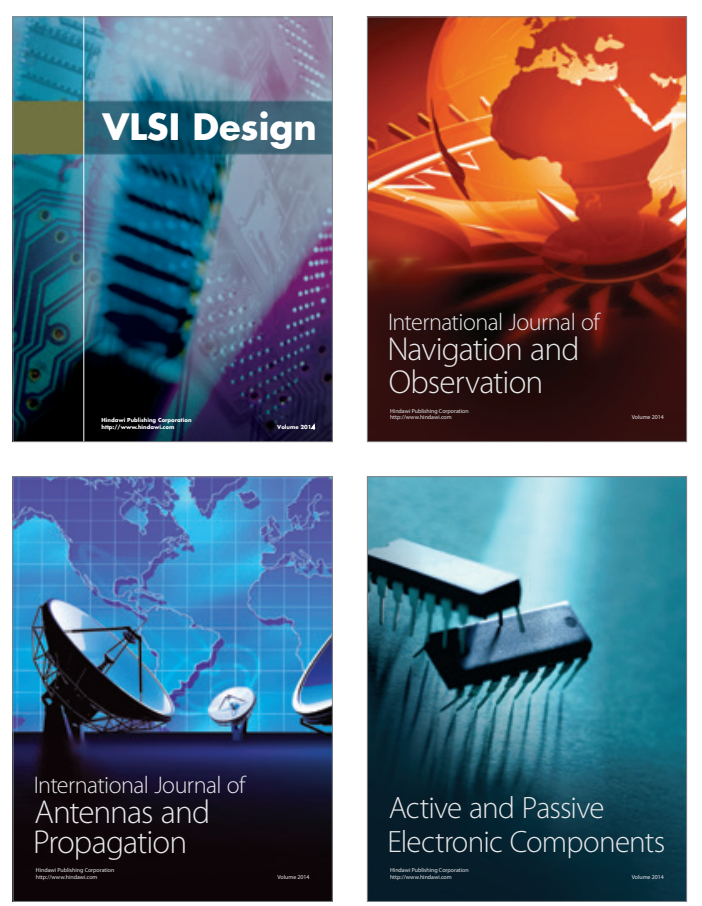
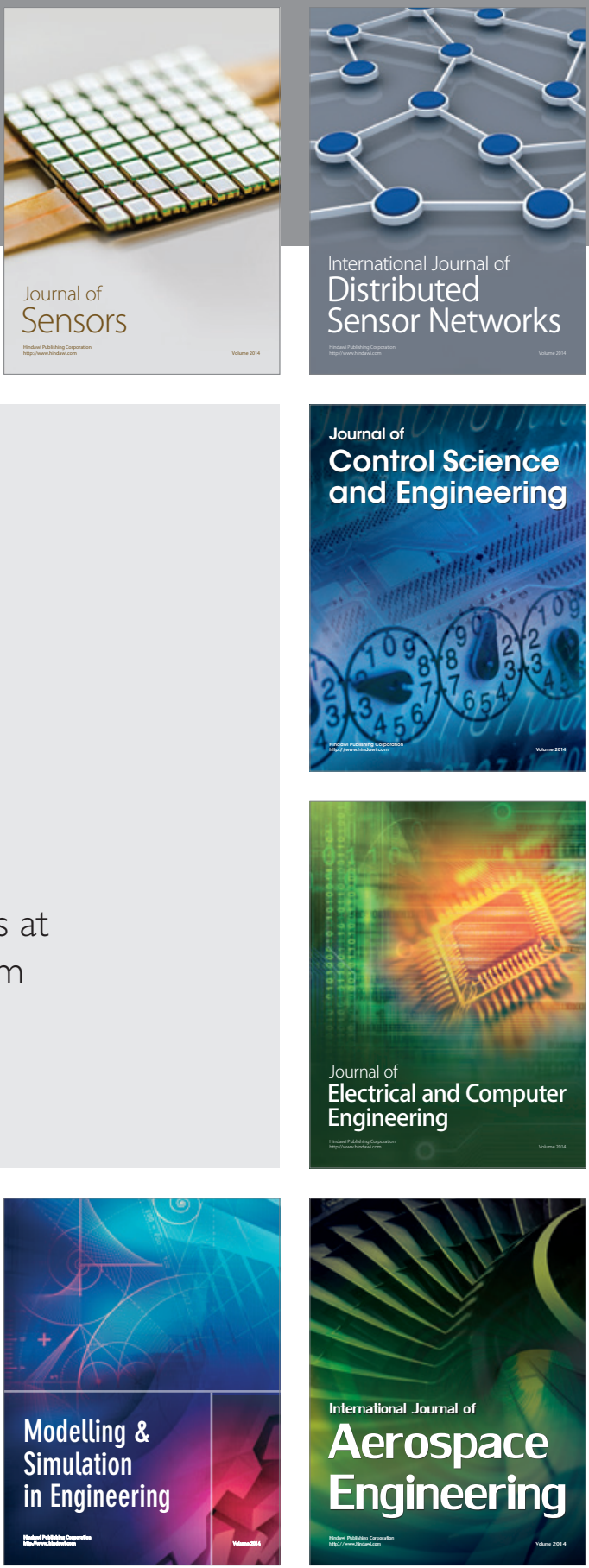

Journal of

Control Science

and Engineering
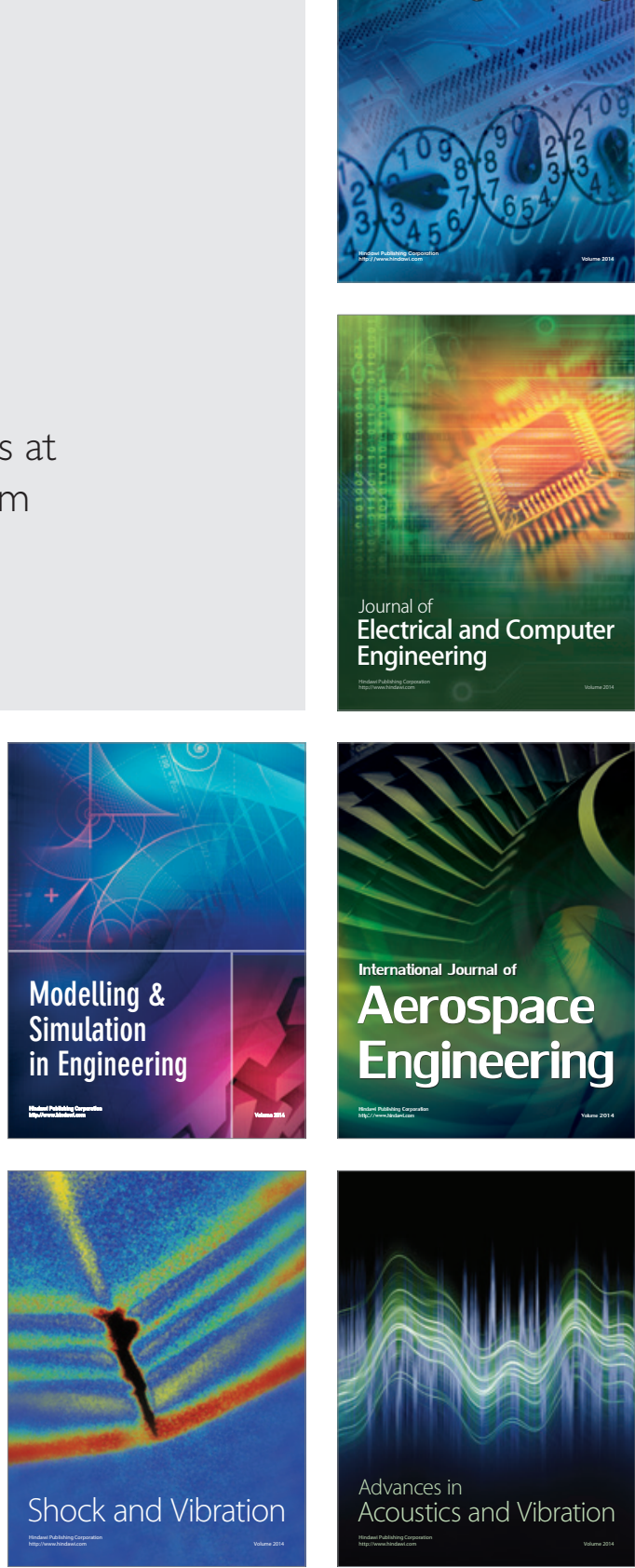